\title{
Mathematische Integrationskurse
}

\author{
Ursula Kowalsky und Karl-Joachim Wirths
}

\begin{abstract}
Integration ist eine Aufgabe unserer Zeit und eine Herausforderung, die in Deutschland aktuell für aufgeregte Diskussionen sorgt. Das Projekt, das dem vorliegenden Artikel zugrunde liegt, ist der Versuch, zum Gelingen von Integration im Rahmen eines Studiums einen Beitrag zu leisten. Das Angebot besteht aus einem einjährigen studienvorbereitenden Intensivkurs, in dem das Erlernen der fehlenden Deutschkenntnisse kombiniert wird mit Einführungen in ein wissenschaftliches Studium. Es richtet sich im Wesentlichen an Geflüchtete, die ein Studium im MINT-Bereich anstreben. Im Besonderen haben wir versucht, die Teilnehmer über mathematische Themen an das Studium heranzuführen. Zum einen bestand die Hoffnung, auf grundlegende Kenntnisse aufbauen zu können, zum anderen gibt es die Erfahrung, dass auch für deutsche Studierende der Ingenieurwissenschaften Mathematik in den ersten Semestern eine nicht zu leugnende Hürde darstellt. Die hier geschilderten Erfahrungen sind nicht uneingeschränkt positiv, jedoch lassen sie die Hoffnung zu, dass Integration auf universitärem Niveau gelingen kann.
\end{abstract}

An der TU Braunschweig gibt es seit April 2016 ein Projekt, das Geflüchtete mit einem in Deutschland anerkannten Abitur auf ein Studium vorbereiten will, indem neben dem Deutschkurs (DSH2) Fachkurse mit Inhalten angeboten werden, die im geplanten Studium von Wichtigkeit sind. Die Finanzierung des Deutschkurses erfolgt mit Drittmitteln des DAAD, wobei die Verwendung der Mittel seitens des Geldgebers an bestimmte Stunden- und Teilnehmeranzahlen gebunden ist. Da die Stundenzahlen über den Umfang des Deutschkurses hinausgehen, sind begleitende Kurse (die ehrenamtlich geleitet werden) Voraussetzung für die Erfüllung der Auflagen des DAAD.

Ausgehend von der Zielrichtung einer Technischen Universität haben wir bevorzugt Bewerberinnen und Bewerber aufgenommen, die am Studium von MINT-Fächern interessiert sind, und haben im April 2016 einen mathematischen Begleitkurs zum Deutschkurs angeboten. Wir haben angenommen, dass wir, unabhängig von der Bedeutung der Mathematik für die MINT-Fächer, auch bei unzureichenden Sprachkenntnissen auf die Formelsprache der Mathematik und dahingehende Vorkenntnisse vertrauen können. Da die Teilnehmerinnen und Teilnehmer fast alle aus Syrien kommen, verfügen sie zudem über Kenntnisse des Englischen. Wir hofften, dass dies ausreichen würde, um mathematische Sachverhalte zu diskutieren und etwas Fachsprache zu lernen.

Als Anreiz wollten wir versuchen, unsere Kursteilnehmer in der Mathematik soweit zu befähigen, wie es das Curriculum eines Studiums des Bauingenieurwesens oder des Maschinenbaus für das erste Semester vorsieht.

Im ersten Halbjahr wurde Analysis unterrichtet, und hier konnte man tatsächlich an vielen Stellen auf vorhandenen Kenntnissen aufbauen und diese im erforderlichen $\mathrm{Maß}$ entwickeln. Die Abschlussklausur nach einem halben Jahr Unterricht bestanden immerhin sechs von 25 Teilnehmern.
Im zweiten Halbjahr haben wir uns dann mit Geometrie und linearer Algebra beschäftigt. Zum ersten Kurs kamen 25 neue Teilnehmer aus einem zweiten Kurs, der im Oktober startete, zusätzlich hinzu. Unsere Hoffnung war, dass die fortgeschrittenen den neuen Teilnehmern helfen würden. Leider blieben die Teilnehmer des ersten Kurses zunehmend dem Unterricht fern. Als Grund wurden vergrößerte Schwierigkeiten beim Lernen der deutschen Sprache angegeben. Tatsächlich gab man aber nach dem Ende des Kurses zu, dass man in Syrien daran gewöhnt sei, zum Lernen gezwungen zu werden. Da wir aus rechtlichen Gründen auf Zwangsmaßnahmen verzichten mussten, ließ sich der oben geschilderte Plan, das Lernziel des ersten Semesters eines Ingenieurstudiums zu erreichen, nicht verwirklichen.

Eine zusätzliche Schwierigkeit war der von uns nicht vorhergesehene Umstand, dass Geometrie und lineare Algebra weit stärker als Analysis auf das richtige Verständnis der Sprache angewiesen sind. Es ist uns beispielsweise nicht gelungen, die Begriffe der linearen Unabhängigkeit oder des Eigenvektors hinreichend zu vermitteln. Auch wurde uns berichtet, dass diese Fächer in Syrien für weniger wichtig als Analysis erachtet würden. Die zugehörige Klausur hat dann auch nur ein Teilnehmer bestanden, der allerdings schon an einem englischsprachigen Universitätsunterricht teilgenommen hatte.

Immerhin haben zehn unserer Teilnehmer nach einem Jahr das DSH2-Zertifikat erhalten und jetzt zum Teil ein Studium aufgenommen. Bei diesen haben wir keine Zweifel daran, dass sie ein Studium in Deutschland erfolgreich absolvieren werden. Sieben weitere Teilnehmer haben die Deutschprüfung mit dem DSH1-Zertifikat abgeschlossen und versuchen jetzt, in einem Wiederholungskurs die zum Bestehen mit DSH2-Zertifikat erforderlichen Kenntnisse zu erwerben. Auch bei ihnen sehen wir gute Aussichten für ein Studium. 
